\title{
A Computer Simulation of the EPI Survey Strategy
}

\author{
STANLEY LEMESHOW*, ALEXANDRE G TSERKOVNYI**, \\ JAMES LEONARD TULLOCH $\dagger$, JOHN E DOWD $\ddagger$, STEVEN K LWANGA‡ \\ AND JACOBUS KEJAS
}

\begin{abstract}
Lemeshow S (Division of Public Health, School of Health Sciences, University of Massachusetts, Amherst MA 01003, USA), Tserkovnyi A G, Tulloch J L, Dowd J E, Lwanga S K and Keja J. A computer simulation of the EPI survey strategy. International Journal of Epidemiology 1985, 14: 473-481.

A Monte Carlo simulation study was designed to evaluate the sample survey technique currently used by the Expanded Programme on Immunization (EPI) of the World Health Organization. Of particular interest was how the EPI strategy compared to a more traditional sampling strategy with respect to bias and variability of estimates. It was also of interest to investigate whether the estimates of population vaccination coverage were accurate to within 10 percentage points of the actual levels. It was found that within particular clusters, the EPI method was particularly sensitive to pocketing of vaccinated individuals, but the more traditional method gave more accurate and less variable results under a variety of conditions. However, the stated goal of the EPI, of being able to produce population estimates accurate to within 10 percentage points of the true levels in the population, was satisfied in the artificially created populations studied.
\end{abstract}

The Expanded Programme on Immunization (EPI) of the World Health Organization continually faces the problem of documenting disease-specific morbidity, mortality and immunization coverage rates in developing countries. Since this information is not readily available to local health managers, EPI has worked to develop a method which could obtain accurate information quickly and cheaply and, in addition, could be implemented in a relatively standardized manner from one country to another.

The sampling scheme used by EPI may be characterized as a probability proportionate to size (PPS) cluster sample. Contrary to classical statistical theory, however, and because of logistic and managerial constraints in field operations, the EPI strategy is to select only the first household within a cluster at random. This household $(\mathrm{HH})$ is visited to determine if any children in the target age group live there and, if so, to collect the required information. If no child of the right age lives in the first $\mathrm{HH}$ visited, the interviewer

\footnotetext{
- Division of Public Health, School of Health Sciences, University of Massachusetts, Amherst, MA 01003, USA.

** Division of Information Systems Support, World Health Organization, 1211 Geneva, Switzerland.

$\dagger$ Diarrhoeal Diseases Control Program, World Health Organization, 1211 Geneva, Switzerland.

\# Epidemiology and Statistical Methodology Unit, World Health Organization, 1211 Geneva, Switzerland.

\& Expanded Programme on Immunization, World Health Organization, 1211 Geneva, Switzerland.
}

proceeds to the 'physically closest' HH. This process continues until seven children are identified within a cluster. Details of the EPI sampling procedure have been presented elsewhere. ${ }^{1-3}$

This paper describes the results of a Monte Carlo simulation study which evaluated the EPI survey methodology in three ways:

(1) By assessing its ability to achieve the stated objective of producing estimates of population vaccination coverage accurate to within 10 percentage points of the actual level;

(2) By examining its performance relative to a classical PPS sampling strategy with respect to bias and variability of estimates of true immunization coverage;

(3) By identifying those factors which may produce high bias and variability at the individual cluster level.

\section{THE SIMULATION DESIGN}

Thirty artificially constructed population clusters were established. Each cluster had a specific combination of population density, household distribution pattern, immunization rate, and immunization pattern.

\section{Population Density}

Each cluster had $600 \mathrm{HH}$; one in every seven $\mathrm{HH}$ contained a child in the target age group. Population density within the cluster was controlled by using matrices into which the $\mathrm{HH}$ were placed. Four density types were defined, ranging from 'low' representing sparsely populated areas $(9.4 \mathrm{HH} / 100$ cells) to 'very 
high' representing highly urbanized areas $(66.7 \mathrm{HH} /$ 100 cells).

\section{Household Distribution Pattern}

Four basic $\mathrm{HH}$ distribution patterns were adopted. In the first, $\mathrm{HH}$ were distributed at random. In the second, cells in the matrix close to an assumed central road or stream had a higher probability of containing a $\mathrm{HH}$ than those furthest away. In the third, $\mathrm{HH}$ were concentrated around a single focus (such as a church, town hall, or market). The fourth pattern reflected a community with two central foci. Probabilities of $\mathrm{HH}$ placement were created for each of the four density types.

\section{Placement of Children}

One out of every seven $\mathrm{HH}$ contained a child. This placement was done randomly.

\section{Level of Coverage}

The proportion of children within a particular cluster specified to be vaccinated was fixed at one of five levels: $10 \%, 30 \%, 50 \%, 70 \%$, or $90 \%$.

\section{Identification of Vaccinated Children}

One of four possible strategies was used to determine which of the children in a cluster were vaccinated. In the first, vaccinated children were identified at random among all children in a particular cluster at the specified level of coverage. The second strategy established a single 'pocket of vaccination' by randomly identifying a $\mathrm{HH}$ containing a child, vaccinating that child, and then, using that $\mathrm{HH}$ as a focus, all children identified around it were designated to be vaccinated. This process continued until the required number of vaccinated children was achieved. The third strategy established two pockets of vaccination. The fourth strategy established fjve pockets of vaccination, each containing $20 \%$ of the total number of children specified to be vaccinated.

\section{DESIGN OF THE SIMULATION Random Number Generation}

Uniform $[0,1)$ random numbers were used to construct clusters with specific combinations of parameters, and to simulate the two sampling strategies. The random number generator used is based on the algorithm described by Lewis et al, ${ }^{4}$ and the required seeds were drawn from the PL/1 TIME function.

\section{Creation of the Clusters}

Once the particular parameters of the cluster were specified, there were known probabilities associated with each of the cells in the matrix with respect to the existence of a $\mathrm{HH}$ and a child. Starting with the upper left-hand cell of the matrix, a random number was generated and compared with the corresponding probability of a $\mathrm{HH}$. If the number generated was less than the probability of a $\mathrm{HH}$ in the particular cell, a $\mathrm{HH}$ was placed. If the $\mathrm{HH}$ was placed, a second random number was generated in order to determine whether it contained a child. If this random number was less than 0.1428 (corresponding to $1 / 7$ probability of a child irrespective of the population density), a child was placed. This process was repeated for every cell in the matrix. Following $\mathrm{HH}$ and child placement, vaccinated children were identified in accordance with the specified immunization pattern and level of coverage. If the vaccination pattern was to be uniform, a random number was generated for each cell containing a child. If this number was less than the specified coverage rate for that cluster, the child was identified as 'vaccinated'. For clusters with vaccination pockets, a random coordinate of the matrix was generated. If this cell contained a child, that child was vaccinated and the cell became the focus of a 'pocket of vaccination'. If that cell did not contain a child, new random coordinates were generated until a child was found. This process of forming the pockets of vaccination was repeated until the specified number of pockets was created.

\section{Household Visitation-the EPI and SRS Algorithms}

To simulate the process of identifying the 'physically closest' $\mathrm{HH}$ as in the EPI methodology, a cell with a $\mathrm{HH}$ was randomly selected in the matrix. This $\mathrm{HH}$ became the starting point for $\mathrm{HH}$ visits. If the $\mathrm{HH}$ did not contain a child, the eight cells surrounding the focal cell were searched for a $\mathrm{HH}$, proceeding counter-clockwise beginning with the cell in the lower right-hand corner. If no $\mathrm{HH}$ existed in these cells, the radius was increased and searched in the same manner. The process was terminated when the first $\mathrm{HH}$ was detected. If a child was present, vaccination status was recorded; otherwise, the next closest $\mathrm{HH}$ was sought using the algorithm described above, until seven children were identified and their vaccination status recorded. In contrast, the SRS strategy used seven randomly selected starting points in each cluster, and nearest $\mathrm{HH}$ were visited until a single child's vaccination status was recorded.

\section{The Simulation Process}

Once a cluster was established with its particular placement of $\mathrm{HH}$, vaccinated and unvaccinated children, 500 independent samples of seven children 
each were selected using the EPI sampling strategy and 500 additional independent samples were selected using the SRS strategy. Each sample resulted in an estimate of the proportion of children actually vaccinated in the entire cluster. Let $\widetilde{E}\left(\hat{P}_{E_{i}}\right)$ denote the average of the 500 estimated proportions resulting from the EPI strategy. used in the $\mathrm{i}$-th cluster, and let $\widetilde{\mathrm{E}}\left(\hat{\mathrm{P}}_{\mathrm{S}_{\mathrm{i}}}\right)$ denote the corresponding average with the SRS method. The following outcome measures were computed for the $\mathrm{i}$-th cluster:

$$
\begin{aligned}
& \operatorname{BIÃS}\left(\hat{\mathrm{P}}_{E_{\mathrm{i}}}\right)=\left\{\tilde{\mathrm{E}}\left(\hat{\mathrm{P}}_{\mathrm{E}_{\mathrm{j}}}\right)-\mathrm{P}_{\mathrm{i}}\right\} \text { and BIÃS }\left(\hat{\mathrm{P}}_{\mathrm{S}_{\mathrm{i}}}\right)= \\
& \left\{\tilde{E}\left(\hat{P}_{S_{i}}\right)-P_{i}\right\} \\
& \operatorname{VÃR}\left(\hat{\mathrm{P}}_{E_{i}}\right)=\sum_{\mathbf{j}=1}^{500}\left(\hat{\mathrm{P}}_{E_{i j}}-\tilde{E}\left(\hat{\mathrm{P}}_{E_{\mathrm{i}}}\right)\right)^{2} / 500 \text { and } \\
& \operatorname{VÃR}\left(\hat{\mathrm{P}}_{\mathrm{S}_{\mathrm{i}}}\right)=\sum_{j=1}^{500}\left(\hat{\mathrm{P}}_{\mathrm{S}_{\mathrm{j}}}-\tilde{\mathrm{E}}\left(\hat{\mathrm{P}}_{\mathrm{S}_{\mathrm{i}}}\right)\right)^{2} / 500 \\
& \operatorname{MS} E\left(\hat{P}_{E_{i}}\right)=\sum_{j=1}^{500}\left(\hat{P}_{E_{i j}}-P_{i}\right)^{2} / 500 \text { and } M \tilde{S} E\left(\hat{P}_{S_{i}}\right) \\
& 500 \\
& =\sum\left(\hat{\mathbf{P}}_{\mathrm{S}_{\mathrm{ij}}}-\mathrm{P}_{\mathrm{i}}\right)^{2} / 500 \\
& j=1
\end{aligned}
$$

Also computed was the absolute relative bias, defined as

$$
\begin{aligned}
& \operatorname{AR̃B}\left(\hat{\mathrm{P}}_{E_{\mathrm{i}}}\right)=\left|\operatorname{BI} \tilde{A} S\left(\hat{\mathrm{P}}_{E_{\mathrm{i}}}\right)\right| / \mathrm{P}_{\mathrm{i}} \text { and } \mathrm{A} \tilde{\mathrm{R} B}\left(\hat{\mathrm{P}}_{\mathrm{S}_{\mathrm{j}}}\right) \\
& =\left|\operatorname{BIÂS}\left(\hat{\mathrm{P}}_{\mathrm{S}_{\mathrm{i}}}\right)\right| / \mathrm{P}_{\mathrm{i}}
\end{aligned}
$$

where $\mathrm{P}_{\mathrm{i}}$ is the actual proportion of children vaccinated in the $\mathrm{i}$-th cluster. (Note that because a random process was employed in the creation of the clusters, the actual level of vaccination attained was not precisely equal to the level specified, although differences were typically small.)

In order to simulate programme applications of these sampling methods, five populations of 30 clusters were constructed, the parameters of which were chosen to reflect actual population characteristics. The proportion of vaccinated children in the population was estimated using the EPI results as follows:

$$
\hat{P} E=\sum_{i=1}^{30} \hat{P}_{E_{i}} / 30
$$

where $\hat{P}_{E_{j}}$ is the estimated proportion in the i-th cluster using the EPI methodology. Similarly, the estimated proportion using samples selected with SRS was estimated as:

$$
\hat{\mathrm{P}} \mathrm{S}=\sum_{\mathbf{i}=1}^{30} \hat{\mathrm{P}}_{\mathrm{Si}} / 30
$$

These estimates were produced for each of the 500 replications of the process and overall comparative statistics were calculated as follows:

$$
\begin{aligned}
& \mathrm{BI} \tilde{\mathrm{A}} \mathrm{S}_{\mathrm{E}}=\{\tilde{\mathrm{E}}(\hat{\mathrm{P} E})-\mathrm{P}\} \text { and } \mathrm{BI} \tilde{\mathrm{A}} \mathrm{S}_{\mathrm{S}}=\{\tilde{\mathrm{E}}(\hat{\mathrm{P}} \mathrm{S})-\mathrm{P}\} \\
& V \tilde{A} R_{E}=\sum_{i=1}^{500}\left(\hat{P}_{i}^{E}-\tilde{E}\left(\hat{P}^{E}\right)\right)^{2} / 500 \text { and } V \tilde{A} R_{S} \\
& =\sum_{i=1}^{500}\left(\hat{P}_{i}^{S}-\tilde{E}\left(\hat{P}^{S}\right)\right)^{2} / 500 \\
& M \tilde{S} E_{E}=\sum_{i=1}^{500}\left(\hat{P}_{i}^{E}-P\right)^{2} / 500 \text { and } M \tilde{S} E_{S}= \\
& 500 \\
& \sum\left(\hat{\mathrm{P}}_{i}^{S}-\mathrm{P}\right)^{2} / 500 \\
& i=1
\end{aligned}
$$

Note that $M \tilde{S} E_{E}=B I \tilde{A} S_{E}^{2}+V \tilde{A} R_{E}$ and $M \tilde{S} E_{S}=$ $B I \tilde{A} S_{S}{ }^{2}+V \tilde{A} R_{S}$.

It should be emphasized that the term 'bias' is a statistical concept used to compare, in a very specific manner, estimators produced from two sampling designs. It is not used as in epidemiological research (ie, any circumstance which results in obscuring or artificially creating an observed risk factor/disease association).

\section{Limitations}

As with any computer simulation, there were basic limitations which could influence the interpretation of the results. First, while an attempt was made to reflect such population characteristics as density, placement of households, and location and proportion of children vaccinated, it was not possible to incorporate many important, dynamic but unmeasurable qualities which characterize real populations. Second, in actual field operations, non-sampling errors due to inadequate coverage, non-response and interviewer bias are usually considerable. No attempt was made to address these basic problems. Third, simplifications necessary for the simulation could be viewed as exaggerations of reality. 
For example, pocketing of vaccinated children was exaggerated in that there was $100 \%$ coverage within a pocket and $0 \%$ coverage elsewhere. Such extremes would be rare in real populations. Also, the chosen cluster size of $600 \mathrm{HH}$ is larger than many typical villages where EPI surveys might be conducted. Fourth, the algorithms used for simulating the movement from house to house in both survey strategies were based on an orderly arrangement of rows and columns within a grid. This clearly does not reflect actual conditions encountered in the field. Finally, the simulations were quite expensive to perform, with each run of 500 replications within each set of 30 clusters costing approximately $\$ 100$. Therefore, not all combinations of cluster characteristics were used.

\section{RESULTS}

\section{Evaluation Within Five Artificial Populations}

Table 1 presents results for the first of five artificially created populations from which repeated samples were taken carried out in order to evaluate the performance of the EPI and SRS methodologies. Columns 2 through 5 of this table describe the population density and distribution, and the vaccination pattern and coverage for each of the 30 clusters. The performance of the EPI methodology relative to its stated aim of estimation within 10 percentage points is summarized in the data at the bottom of the table. Performance relative to SRS can also be determined from these data and from the 'over all clusters' line in the table. Bias and variability at the cluster level are presented in the last four columns of the body of the table.

The population described in Table 1 was designed to represent a predominantly urban area with high vaccination coverage. Population density, $\mathrm{HH}$ distribution, $\mathrm{HH}$ pattern, and level of vaccination were varied among the 30 clusters, as seen in columns 2 through 5 . As can be seen in column 5 , the proportion of vaccinated children was designed to be quite high in the various clusters and, as seen in column 6 , the actual proportion vaccinated was close to the planned level in most instances. The planned overall vaccination rate was $79 \%$, but a rate of $77.7 \%$ was actually attained. The average of the 500 estimates of the proportions of children vaccinated in each cluster is presented in columns 7 and 8 for the two sampling strategies. Columns 9 and 10 present the bias for both strategies, and columns 11 and 12 present the mean square error (MSE). In addition, the overall proportion vaccinated in the population was estimated from the data collected from all 30 clusters. This was done 500 times for each of the sampling strategies and the mean estimated proportion, the bias and the MSE are presented in the row labelled 'over all clusters'.

From the data at the bottom of Table 1, it can be seen that only one $(0.2 \%)$ of the $500 \mathrm{EPI}$ estimates of the overall vaccination coverage differed from the true level $(P)$ by more than 10 percentage points. None of the 500 SRS estimates differed from the true level to this extent. Using a more stringent requirement that the estimated levels fall within $10 \%$ of $P$, it is seen that six $(1.2 \%)$ of the EPI samples produced excessive values while one $(0.2 \%)$ SRS sample underestimated $P$. Comparing the overall EPI and SRS estimates of $P$ reveals that while the former was somewhat more biased, the two methods produced equivalent MSE. With respect to results at the cluster level, the EPI methodology produced estimates with greater bias than the SRS methodology in 20 of the 30 clusters. The magnitude of the differences in the bias and MSE between the methods was typically small.

A mixed (urban/rural) population with a planned overall vaccination coverage of $46 \%$ and pocketing of vaccination in 16 of the 30 clusters was also used in the simulation. Results are presented in Table 2. EPI estimates of total coverage exceeded the 10 percentage point limit in $18(3.6 \%)$ samples, while SRS produced no estimates with this degree of bias. The narrower limits $(P \pm 0.10(P))$ were exceeded $219(43.8 \%)$ and 117 $(23.4 \%)$ times with the EPI and SRS methods, respectively. The extreme values were typically underestimates. Overall bias and MSE were higher with the EPI method. At the cluster level, the SRS method produced lower bias in 20 clusters. In the clusters with pocketing of vaccination, the EPI method typically had considerably higher bias and MSE.

The three additional populations constructed represented:

(a) a population with both urban and rural clusters and a planned overall vaccination coverage of $65 \%$;

(b) a predominantly rural population with the same planned overall vaccination coverage $(65 \%)$ as in (a);

(c) a largely rural population with low planned overall vaccination coverage $(17 \%)$.

The findings using these three populations closely paralleled those shown in Table 2 and results are not presented here. Clearly, numerous other populations could have been constructed and examined.

\section{Evaluation of the Basic Factors}

An analysis of variance (ANOVA) was designed with the following factors and levels:

$\begin{array}{ll}\text { Factor } & \text { Levels } \\ \text { Density } & \text { Low, Moderate, Very High }\end{array}$


TABLE 1 Cluster parameters, coverage level achieved, mean estimated coverage, bias and mean squared error for EPI versus SRS estimates for vaccination coverage for each of 30 clusters and over all clusters for a predominantly urban area with planned $79 \%$ overall coverage.

\begin{tabular}{|c|c|c|c|c|c|c|c|c|c|c|c|}
\hline \multirow[b]{3}{*}{ Cluster } & \multicolumn{4}{|c|}{ Cluster parameters } & \multirow{3}{*}{$\begin{array}{l}\text { Achieved } \\
\text { coverage }\end{array}$} & \multirow{2}{*}{\multicolumn{2}{|c|}{$\begin{array}{c}\text { Mean } \\
\text { estimated coverage }\end{array}$}} & \multirow{2}{*}{\multicolumn{2}{|c|}{ Bias }} & \multirow{2}{*}{\multicolumn{2}{|c|}{ MSE }} \\
\hline & & Household & Vaccination & Specified & & & & & & & \\
\hline & Density & design & design & level & & EPI & SRS & EPI & SRS & EPI & SRS \\
\hline 1 & Low & Road & Random & 0.30 & 0.267 & 0.270 & 0.276 & 0.003 & 0.009 & 0.024 & 0.024 \\
\hline 2 & Low & Random & Random & 0.50 & 0.518 & 0.512 & 0.515 & -0.006 & -0.003 & 0.017 & 0.031 \\
\hline 3 & Low & Road & Random & 0.50 & 0.424 & 0.402 & 0.441 & -0.220 & 0.017 & 0.028 & 0.034 \\
\hline 4 & Medium & Random & Random & 0.50 & 0.375 & 0.202 & 0.334 & -0.173 & -0.041 & 0.053 & 0.031 \\
\hline 5 & Medium & Road & Random & 0.90 & 0.886 & 0.919 & 0.885 & 0.033 & -0.001 & 0.013 & 0.013 \\
\hline 6 & Medium & Random & Random & 0.90 & 0.876 & 0.897 & 0.865 & 0.021 & -0.011 & 0.010 & 0.013 \\
\hline 7 & Medium & Road & Random & 0.90 & 0.928 & 0.913 & 0.914 & -0.015 & -0.014 & 0.007 & 0.010 \\
\hline 8 & Medium & Random & Random & 0.90 & 0.908 & 0.909 & 0.901 & 0.001 & -0.007 & 0.012 & 0.012 \\
\hline 9 & Medium & Road & 1 pocket & 0.90 & 0.894 & 0.920 & 0.909 & 0.026 & 0.015 & 0.035 & 0.011 \\
\hline 10 & Medium & Random & 2 pockets & 0.90 & 0.891 & 0.895 & 0.906 & 0.004 & 0.015 & 0.043 & 0.012 \\
\hline 11 & Medium & Road & 5 pockets & 0.90 & 0.886 & 0.845 & 0.889 & -0.041 & 0.003 & 0.027 & 0.013 \\
\hline 12 & High & Random & 1 pocket & 0.50 & 0.494 & 0.481 & 0.537 & -0.013 & 0.043 & 0.122 & 0.035 \\
\hline 13 & High & Road & 2 pockets & 0.50 & 0.483 & 0.547 & 0.521 & 0.064 & 0.038 & 0.004 & 0.034 \\
\hline 14 & High & Random & 5 pockets & 0.70 & 0.663 & 0.823 & 0.761 & 0.160 & 0.098 & 0.102 & 0.035 \\
\hline 15 & High & Road & 1 pocket & 0.90 & 0.894 & 0.905 & 0.906 & 0.011 & 0.012 & 0.100 & 0.012 \\
\hline 16 & High & Random & 2 pockets & 0.90 & 0.889 & 0.984 & 0.941 & 0.095 & 0.052 & 0.014 & 0.010 \\
\hline 17 & High & Road & 5 pockets & 0.90 & 0.893 & 0.839 & 0.867 & -0.054 & -0.026 & 0.007 & 0.012 \\
\hline 18 & High & Random & 1 pocket & 0.90 & 0.900 & 0.954 & 0.957 & 0.054 & 0.057 & 0.023 & 0.009 \\
\hline 19 & High & Road & 2 pockets & 0.90 & 0.886 & 0.987 & 0.960 & 0.101 & 0.074 & 0.015 & 0.011 \\
\hline 20 & High & Random & 5 pockets & 0.90 & 0.833 & 0.948 & 0.898 & 0.115 & 0.065 & 0.024 & 0.016 \\
\hline 21 & High & Road & 1 pocket & 0.90 & 0.897 & 0.968 & 0.950 & 0.071 & 0.053 & 0.012 & 0.010 \\
\hline 22 & High & Random & 2 pockets & 0.90 & 0.884 & 0.809 & 0.853 & -0.075 & -0.031 & 0.051 & 0.017 \\
\hline 23 & Very high & 1 centre & Random & 0.50 & 0.542 & 0.535 & 0.438 & -0.007 & -0.104 & 0.012 & 0.035 \\
\hline 24 & Very high & 2 centres & Random & 0.70 & 0.731 & 0.900 & 0.805 & 0.169 & 0.074 & 0.044 & 0.024 \\
\hline 25 & Very high & 1 centre & Random & 0.90 & 0.885 & 0.873 & 0.812 & -0.012 & -0.073 & 0.010 & 0.023 \\
\hline 26 & Very high & 2 centres & Random & 0.90 & 0.879 & 0.827 & 0.799 & -0.052 & -0.080 & 0.018 & 0.022 \\
\hline 27 & Very high & 1 centre & Random & 0.90 & 0.969 & 0.991 & 0.977 & 0.022 & 0.008 & 0.002 & 0.003 \\
\hline 28 & Very high & 2 centres & Random & 0.90 & 0.880 & 0.928 & 0.820 & 0.048 & -0.060 & 0.009 & 0.019 \\
\hline 29 & Very high & 1 centre & Random & 0.90 & 0.867 & 0.896 & 0.878 & 0.029 & 0.011 & 0.005 & 0.014 \\
\hline 30 & Very high & 2 centres & Random & 0.90 & 0.917 & 0.857 & 0.960 & -0.060 & 0.043 & 0.006 & 0.007 \\
\hline Over all clusters & & & & & 0.777 & 0.791 & 0.782 & 0.0014 & 0.0006 & 0.001 & 0.001 \\
\hline
\end{tabular}

Distribution of overall estimates

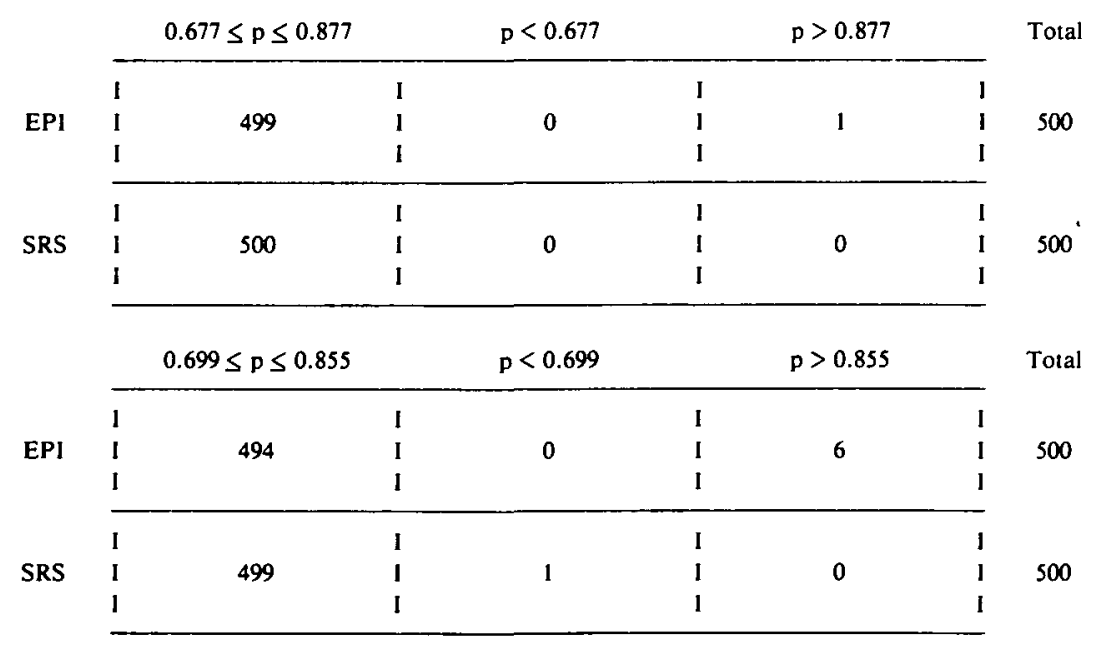


TABLE 2 Cluster parameters, coverage level achieved, mean estimated coverage, bias and mean squared error for EPI versus SRS estimates for vaccination coverage for each of 30 clusters and over all clusters for a mixed (urban/rural) area with planned $46 \%$ overall coverage.

\begin{tabular}{|c|c|c|c|c|c|c|c|c|c|c|c|}
\hline \multirow[b]{3}{*}{ Cluster } & \multicolumn{4}{|c|}{ Cluster parameters } & \multirow{3}{*}{$\begin{array}{l}\text { Achieved } \\
\text { coverage }\end{array}$} & \multirow{2}{*}{\multicolumn{2}{|c|}{$\begin{array}{c}\text { Mean } \\
\text { estimated coverage }\end{array}$}} & \multirow{2}{*}{\multicolumn{2}{|c|}{ Bias }} & \multirow{2}{*}{\multicolumn{2}{|c|}{ MSE }} \\
\hline & \multirow[b]{2}{*}{ Density } & \multirow{2}{*}{$\begin{array}{l}\text { Household } \\
\text { design }\end{array}$} & \multirow{2}{*}{$\begin{array}{l}\text { Vaccination } \\
\text { design }\end{array}$} & \multirow{2}{*}{$\begin{array}{l}\text { Specified } \\
\text { level }\end{array}$} & & & & & & & \\
\hline & & & & & & EPl & SRS & EPI & SRS & EPl & SRS \\
\hline 1 & Low & Road & Random & 0.30 & 0.304 & 0.248 & 0.273 & -0.056 & -0.031 & 0.024 & 0.028 \\
\hline 2 & Low & Random & Random & 0.10 & 0.081 & 0.097 & 0.065 & 0.016 & -0.016 & 0.008 & 0.008 \\
\hline 3 & Low & Road & Random & 0.10 & 0.078 & 0.067 & 0.099 & -0.011 & 0.021 & 0.007 & 0.011 \\
\hline 4 & Low & Random & Random & 0.10 & 0.141 & 0.169 & 0.174 & 0.028 & 0.033 & 0.017 & 0.018 \\
\hline 5 & Low & Road & Random & 0.10 & 0.154 & 0.130 & 0.132 & -0.024 & -0.022 & 0.011 & 0.014 \\
\hline 6 & Medium & Random & Random & 0.10 & 0.118 & 0.070 & 0.079 & -0.048 & -0.039 & 0.016 & 0.012 \\
\hline 7 & Medium & Road & Random & 0.10 & 0.082 & 0.094 & 0.107 & 0.012 & 0.025 & 0.006 & 0.012 \\
\hline 8 & Medium & Random & 1 pocket & 0.30 & 0.291 & 0.419 & 0.305 & 0.128 & 0.014 & 0.153 & 0.031 \\
\hline 9 & Medium & Road & 2 pockets & 0.30 & 0.299 & 0.308 & 0.319 & 0.009 & 0.020 & 0.107 & 0.027 \\
\hline 10 & Medium & Random & 5 pockets & 0.30 & 0.298 & 0.483 & 0.285 & 0.185 & -0.013 & 0.124 & 0.024 \\
\hline 11 & Medium & Road & 1 pocket & 0.30 & 0.299 & 0.136 & 0.195 & -0.163 & -0.104 & 0.097 & 0.032 \\
\hline 12 & Medium & Random & 2 pockets & 0.50 & 0.500 & 0.351 & 0.461 & -0.149 & -0.039 & 0.136 & 0.035 \\
\hline 13 & Medium & Road & 5 pockets & 0.50 & 0.460 & 0.347 & 0.340 & -0.113 & -0.120 & 0.088 & 0.042 \\
\hline 14 & Medium & Random & 1 pocket & 0.50 & 0.500 & 0.292 & 0.361 & -0.208 & -0.139 & 0.185 & 0.052 \\
\hline 15 & High & Road & 2 pockets & 0.30 & 0.295 & 0.487 & 0.344 & 0.192 & 0.049 & 0.139 & 0.034 \\
\hline 16 & High & Random & 5 pockets & 0.30 & 0.241 & 0.346 & 0.250 & 0.105 & 0.009 & 0.087 & 0.023 \\
\hline 17 & High & Road & 1 pocket & 0.30 & 0.293 & 0.047 & 0.181 & -0.246 & -0.112 & 0.078 & 0.033 \\
\hline 18 & High & Random & 2 pockets & 0.50 & 0.500 & 0.355 & 0.477 & -0.145 & -0.023 & 0.115 & 0.031 \\
\hline 19 & High & Road & 5 pockets & 0.50 & 0.460 & 0.193 & 0.359 & -0.267 & -0.101 & 0.116 & 0.036 \\
\hline 20 & High & Random & 1 pocket & 0.50 & 0.500 & 0.401 & 0.530 & -0.099 & 0.030 & 0.102 & 0.035 \\
\hline 21 & High & Road & 2 pockets & 0.50 & 0.494 & 0.109 & 0.340 & -0.385 & -0.154 & 0.182 & 0.052 \\
\hline 22 & High & Random & 5 pockets & 0.50 & 0.455 & 0.669 & 0.511 & 0.214 & 0.056 & 0.112 & 0.032 \\
\hline 23 & High & Road & Random & 0.90 & 0.920 & 0.989 & 0.970 & 0.069 & 0.050 & 0.006 & 0.006 \\
\hline 24 & High & Random & Random & 0.90 & 0.896 & 0.886 & 0.934 & -0.010 & 0.038 & 0.007 & 0.010 \\
\hline 25 & High & Road & Random & 0.90 & 0.948 & 0.964 & 0.927 & 0.016 & -0.021 & 0.004 & 0.008 \\
\hline 26 & Very high & 1 centre & 1 pocket & 0.30 & 0.291 & 0.169 & 0.150 & -0.122 & -0.141 & 0.034 & 0.036 \\
\hline 27 & Very high & 1 centre & Random & 0.90 & 0.904 & 0.880 & 0.908 & -0.024 & 0.004 & 0.004 & 0.011 \\
\hline 28 & Very high & 2 centres & Random & 0.90 & 0.896 & 0.911 & 0.919 & 0.015 & 0.023 & 0.021 & 0.010 \\
\hline 29 & Very high & 2 centres & Random & 0.90 & 0.903 & 0.938 & 0.929 & 0.035 & 0.026 & 0.006 & 0.010 \\
\hline 30 & Very high & 2 centres & Random & 0.70 & 0.597 & 0.664 & 0.558 & 0.067 & -0.039 & 0.028 & 0.025 \\
\hline Over all & & & & & 0.435 & 0.407 & 0.416 & -0.027 & -0.018 & 0.002 & 0.001 \\
\hline
\end{tabular}

Distribution of overall estimates

\begin{tabular}{|c|c|c|c|c|c|c|c|c|}
\hline & & $\leq p \leq$ & & $p<0.335$ & & 0.53 & & Total \\
\hline & I & & I & & I & & I & \\
\hline EPI & I & 482 & I & 17 & I & 1 & i & 500 \\
\hline & I & & I & & I & & I & \\
\hline & I & & 1 & & I & & 1 & \\
\hline SRS & I & 500 & 1 & 0 & I & 0 & I & 500 \\
\hline & 1 & & I & & I & & I & \\
\hline & & $\leqslant p \leqslant$ & & $p<0.392$ & & 0.4 & & Total \\
\hline & 1 & & I & & I & & I & \\
\hline EPI & I & 281 & I & 199 & 1 & 20 & 1 & 500 \\
\hline & I & & I & & I & & I & \\
\hline & I & & I & & I & & 1 & \\
\hline SRS & 1 & 383 & $\mathbf{I}$ & 114 & I & 3 & I & 500 \\
\hline & 1 & & I & & I & & 1 & \\
\hline
\end{tabular}


Household Design

Vaccination Design

Vaccination Level

Method

Road, One Centre, Random

Random, One Pocket

$30 \%, 50 \%, 70 \%$

EPI, SRS

Note that while all available factors are included in this ANOVA, the number of levels used is less than the total number available and described earlier in this paper. This was done to limit the expense of the necessary simulations. With the above levels, there were 108 unique combinations of factors and two replications were performed for each such combination. Each replication involved sampling 500 times from a cluster defined by the unique combination of factor levels and computing one of the outcome variables (ie, absolute relative bias or mean square error). Each of the main effects and many of the interactions were significant with respect to absolute relative bias. Higher density clusters demonstrated higher absolute relative bias. Also, while EPI resulted in higher absolute relative bias than did SRS, the trend of increasing absolute relative bias with increasing density which was noted with EPI was not noted with SRS. This resulted in a significant method-by-density interaction. There was a tendency for absolute relative bias to decrease with increasing coverage with both the EPI and SRS methods. As a result, a significant interaction was not observed. There was a very strong main effect due to vaccination design, with pocketing of vaccinated children demonstrating much higher absolute relative bias than random assignment of vaccinated children. The vaccination design by method interaction was also highly significant. This was due to the fact that both the SRS and EPI methods had comparable bias with random vaccination designs, but the EPI method had much greater bias when there was pocketing of vaccination. Examination of the interaction between pocketing and method at different levels of density showed that the tendency for absolute relative bias to be much higher with the EPI method than with the SRS method, when pocketing exists, holds within each of the three density types considered.

Comparable results were observed using MSE as the dependent variable in the ANOVA. Highly significant main effects due to vaccination design, level of coverage, and method were noted. Much higher MSE was observed with the EPI method than with the SRS method and pocketing of vaccinated children resulted in higher MSE than with randomly placed vaccinated children. The only striking interaction was the one between vaccination design and method. When vaccinated children were randomly placed, there was little difference between the methods; however, when pocketing existed, the EPI method demonstrated strikingly higher MSE. The difference between the two methods in the face of pocketing was particularly large in low density clusters. Irrespective of density, there was little difference between the methods if vaccinated children were distributed at random.

\section{DISCUSSION}

The stated aim of the EPI survey methodology is to produce estimates of vaccination coverage accurate to within 10 percentage points of the true level in the population with $95 \%$ confidence. Based on the results from the five simulated populations, there is no evidence to suggest that this aim is not being achieved. Even when more stringent requirements of precision were specified, the EPI method rarely overestimated the actual population proportion (ie, the estimated proportion was rarely greater than $(P \pm 0.10(P))$. Clearly, for programme management purposes, a survey methodology with a high risk of significant overestimation would not be acceptable.

The comparison of the EPI and the more classical SRS strategies demonstrated that the SRS method was superior to the EPI method with respect to bias and MSE, as well as the proportion of times the estimates fell outside of a defined range. Feasibility permitting, the SRS strategy would be the optimal choice; however, operational constraints often override statistical considerations and lead to the selection of the EPI methodology for surveys of vaccination coverage. Given the stated objectives of the EPI survey, this would appear to be a reasonable choice.

The analysis of variance clearly demonstrated that pocketing of vaccinated children in a cluster was closely associated with high bias and variability at the cluster level when the EPI strategy was employed. It should be emphasized that, in any cluster sampling technique, it is inappropriate to produce estimates for individual clusters. Clearly, users of EPI survey results should not succumb to the temptation of using data from individual clusters to draw conclusions concerning levels of coverage in localized areas. This risk would be minimized if names of the particular clusters selected were omitted in the presentation of results.

The reader should not assume that the EPI methodology will always satisfy its stated objective with respect to accuracy. To demonstrate this, a 'worstcase' population was constructed which combined high population density, low vaccination coverage and pocketing of vaccination in all clusters. Table 3 presents the results for this population in a format similar to that used in Tables 1 and 2. Use of the EPI method resulted in estimates of acceptable precision $( \pm$ 10 percentage points) in only $155(31 \%)$ of the 500 
TABLE 3 Cluster parameters, coverage level achieved, mean estimated coverage, bias and mean squared error for EPI versus SRS estimates for vaccination coverage for each of 30 clusters and over all clusters for a worst case population having a planned $30 \%$ overall coverage.

\begin{tabular}{|c|c|c|c|c|c|c|c|c|c|c|c|}
\hline \multirow[b]{3}{*}{ Cluster } & \multicolumn{4}{|c|}{ Cluster parameters } & \multirow{3}{*}{$\begin{array}{l}\text { Achieved } \\
\text { coverage }\end{array}$} & \multirow{2}{*}{\multicolumn{2}{|c|}{$\begin{array}{c}\text { Mean } \\
\text { estimated coverage }\end{array}$}} & \multirow{2}{*}{\multicolumn{2}{|c|}{ Bias }} & \multirow{2}{*}{\multicolumn{2}{|c|}{ MSE }} \\
\hline & & Household & Vaccination & Specified & & & & & & & \\
\hline & Density & design & design & level & & EPI & SRS & EPI & SRS & EPI & SRS \\
\hline 1 & High & 1 centre & 1 pocket & 0.30 & 0.293 & 0.096 & 0.275 & -0.197 & -0.018 & 0.058 & 0.023 \\
\hline 2 & High & 1 centre & 1 pocket & 0.30 & 0.292 & 0.041 & 0.153 & -0.251 & -0.139 & 0.075 & 0.038 \\
\hline 3 & High & 1 centre & 1 pocket & 0.30 & 0.289 & 0.072 & 0.227 & -0.217 & -0.062 & 0.067 & 0.026 \\
\hline 4 & High & 1 centre & 1 pocket & 0.30 & 0.294 & 0.183 & 0.247 & -0.111 & -0.047 & 0.087 & 0.027 \\
\hline 5 & High & 1 centre & 1 pocket & 0.30 & 0.293 & 0.692 & 0.269 & 0.399 & -0.024 & 0.040 & 0.025 \\
\hline 6 & High & 1 centre & 1 pocket & 0.30 & 0.299 & 0.307 & 0.368 & 0.008 & 0.069 & 0.078 & 0.033 \\
\hline 7 & High & 1 centre & 1 pocket & 0.30 & 0.293 & 0.427 & 0.477 & 0.134 & 0.184 & 0.068 & 0.061 \\
\hline 8 & High & 1 centre & 1 pocket & 0.30 & 0.299 & 0.051 & 0.128 & -0.248 & -0.171 & 0.079 & 0.045 \\
\hline 9 & High & 1 centre & 1 pocket & 0.30 & 0.299 & 0.087 & 0.205 & -0.212 & -0.094 & 0.069 & 0.029 \\
\hline 10 & High & 1 centre & 1 pocket & 0.30 & 0.297 & 0.081 & 0.262 & -0.216 & -0.035 & 0.066 & 0.028 \\
\hline 11 & High & 1 centre & 1 pocket & 0.30 & 0.293 & 0.052 & 0.140 & -0.241 & -0.153 & 0.078 & 0.038 \\
\hline 12 & High & 1 centre & 1 pocket & 0.30 & 0.296 & 0.049 & 0.179 & -0.247 & -0.117 & 0.072 & 0.034 \\
\hline 13 & High & 1 centre & 1 pocket & 0.30 & 0.291 & 0.635 & 0.515 & 0.344 & 0.224 & 0.018 & 0.082 \\
\hline 14 & High & I centre & 1 pocket & 0.30 & 0.292 & 0.444 & 0.375 & 0.152 & 0.083 & 0.025 & 0.034 \\
\hline 15 & High & 1 centre & 1 pocket & 0.30 & 0.290 & 0.118 & 0.169 & -0.172 & -0.121 & 0.061 & 0.034 \\
\hline 16 & Very high & I centre & 1 pocket & 0.30 & 0.288 & 0.131 & 0.150 & -0.157 & -0.138 & 0.056 & 0.037 \\
\hline 17 & Very high & 1 centre & 1 pocket & 0.30 & 0.292 & 0.059 & 0.192 & -0.233 & -0.100 & 0.073 & 0.031 \\
\hline 18 & Very high & 1 centre & 1 pocket & 0.30 & 0.293 & 0.429 & 0.384 & 0.199 & 0.091 & 0.050 & 0.042 \\
\hline 19 & Very high & 1 centre & 1 pocket & 0.30 & 0.297 & 0.047 & 0.183 & -0.250 & -0.114 & 0.073 & 0.033 \\
\hline 20 & Very high & I centre & 1 pocket & 0.30 & 0.290 & 0.055 & 0.218 & -0.235 & -0.072 & 0.067 & 0.027 \\
\hline 21 & Very high & I centre & 1 pocket & 0.30 & 0.289 & 0.070 & 0.217 & -0.219 & -0.072 & 0.065 & 0.028 \\
\hline 22 & Very high & I centre & 1 pocket & 0.30 & 0.295 & 0.046 & 0.166 & -0.249 & -0.129 & 0.074 & 0.035 \\
\hline 23 & Very high & I centre & 1 pocket & 0.30 & 0.297 & 0.392 & 0.238 & 0.095 & -0.059 & 0.101 & 0.025 \\
\hline 24 & Very high & 1 centre & 1 pocket & 0.30 & 0.297 & 0.048 & 0.185 & -0.249 & -0.112 & 0.074 & 0.032 \\
\hline 25 & Very high & 1 centre & 1 pocket & 0.30 & 0.292 & 0.151 & 0.217 & -0.141 & -0.075 & 0.081 & 0.029 \\
\hline 26 & Very high & 1 centre & 1 pocket & 0.30 & 0.296 & 0.045 & 0.167 & -0.251 & -0.129 & 0.074 & 0.035 \\
\hline 27 & Very high & 1 centre & 1 pocket & 0.30 & 0.293 & 0.097 & 0.268 & -0.196 & -0.025 & 0.060 & 0.025 \\
\hline 28 & Very high & I centre & 1 pocket & 0.30 & 0.295 & 0.165 & 0.280 & -0.130 & -0.015 & 0.062 & 0.024 \\
\hline 29 & Very high & I centre & 1 pocket & 0.30 & 0.282 & 0.042 & 0.169 & -0.247 & -0.120 & 0.070 & 0.031 \\
\hline 30 & Very high & 1 centre & 1 pocket & 0.30 & 0.294 & 0.041 & 0.214 & -0.253 & -0.080 & 0.073 & 0.027 \\
\hline Over all clusters & & & & & 0.294 & 0.174 & 0.241 & -0.119 & -0.052 & 0.016 & 0.003 \\
\hline
\end{tabular}

\begin{tabular}{|c|c|c|c|c|c|c|c|c|}
\hline \multicolumn{9}{|c|}{ Distribution of overall estimates } \\
\hline & \multicolumn{2}{|c|}{$0.194 \leq \mathrm{p} \leq 0.394$} & & \multicolumn{2}{|l|}{$p<0.194$} & \multicolumn{2}{|c|}{$\mathrm{p}>0.394$} & Total \\
\hline & I & & I & & I & & 1 & \\
\hline \multirow[t]{3}{*}{ EPI } & 1 & 155 & 1 & 349 & I & 0 & I & 500 \\
\hline & 1 & & I & & 1 & & I & \\
\hline & I & & I & & I & & I & \\
\hline \multirow[t]{4}{*}{ SRS } & 1 & 481 & 1 & 19 & 1 & 0 & 1 & 500 \\
\hline & 1 & & I & & 1 & & I & \\
\hline & & $\leq p \leq 0.323$ & & $p<0.265$ & & 0.323 & & Total \\
\hline & 1 & & I & & I & & I & \\
\hline \multirow[t]{3}{*}{ EPI } & 1 & 4 & I & 496 & I & 0 & I & 500 \\
\hline & 1 & & 1 & & I & & I & \\
\hline & 1 & & 1 & & 1 & & 1 & \\
\hline \multirow[t]{2}{*}{ SRS } & I & 112 & 1 & 388 & 1 & 0 & 1 & 500 \\
\hline & I & & I & & I & & 1 & \\
\hline
\end{tabular}


replications as compared with $481(96.2 \%)$ of the SRS estimates. For both methods, all extreme results were underestimates. Under the more stringent accuracy requirements, neither method would be acceptable. It is not surprising that in this worst-case situation, the EPI estimates were significantly more biased and variable than were the SRS estimates. It should be stressed that it is extremely unlikely that any real population would parallel the one represented by this artificial situation.

This study would appear to provide reassuring evidence to users of EPI surveys, that it is possible to estimate the true level of vaccination coverage to within 10 percentage points in typical populations. It also sets forth a warning to those users who might make inferences about vaccination coverage in individual clusters or groups of clusters. Such disaggregation of the survey data is inadvisable.

\section{REFERENCES}

1 Henderson R H, Sundaresan T. Cluster sampling 10 assess immunization coverage: a review of experience with a simplified sampling method. Bull WHO 1982; 60: 253-60.

2 World Health Organization Expanded Programme on Immunization. Training manual for mid-level managers: evaluate vaccination coverage.

${ }^{3}$ Lemeshow S, Robinson D. Surveys to measure programme coverage and impact: a review of the methodology used by the Expanded Progamme on Immunization. World Health Stat $Q$ 1985; 38: 65-75.

${ }^{4}$ Lewis P A W, Goodman A F, Miller J M. A pseudo random number generator for the System 360. IBM Systems Journal 1969; 8: 136-46.

(Revised version received January 1985) 